\title{
Control of radial propagation and polarity in a plasma jet in surrounding $\mathrm{Ar}$
}

\author{
W. Gong, ${ }^{1,2}$ Y. Yue,${ }^{1,3}$ F. Ma, ${ }^{4}$ F. Yu, ${ }^{1}$ J. Wan,${ }^{1}$ L. Nie, ${ }^{1}$ K. Bazaka, ${ }^{5,6}$ Y. Xian,,${ }^{1,5,6, a)}$ X. Lu, ${ }^{1}$ \\ and K. Ostrikov ${ }^{5,6}$ \\ ${ }^{1}$ State Key Laboratory of Advanced Electromagnetic Engineering and Technology, School of Electrical \\ and Electronic Engineering, Huazhong University of Science and Technology, Wuhan, Hubei 430074, \\ People's Republic of China \\ ${ }^{2}$ National Demonstration Center for Experimental Electrical and Electronic Education, Yangtze University, \\ Jingzhou, Hubei 434000, People's Republic of China and Electronics and Information School, Yangtze \\ University, Jingzhou, Hubei 434000, People's Republic of China \\ ${ }^{3}$ Clean Combustion Research Center, King Abdullah University of Science and Technology, \\ Thuwal 23955-6900, Saudi Arabia \\ ${ }^{4}$ Guangxi Medical Equipment Testing Center, Xinmin Road 1-1, Nanning, Guangxi 530021, \\ People's Republic of China \\ ${ }^{5}$ School of Chemistry, Physics, Mechanical Engineering, Queensland University of Technology, Brisbane, \\ QLD 4000, Australia \\ ${ }^{6}$ CSIRO-QUT Joint Sustainable Processes and Devices Laboratory, Commonwealth Scientific and Industrial \\ Research Organization, PO Box 218, Lindfield, NSW 2070, Australia
}

(Received 28 October 2017; accepted 19 December 2017; published online 8 January 2018)

In recent years, the use of shielding gas to prevent the diffusion of the ambient air, particularly oxygen and nitrogen species, into the effluent of the atmospheric pressure plasma jet, and thus control the nature of chemical species used in the plasma treatment has increased. In this paper, the radial propagation of a plasma jet in ambient Ar is examined to find the key determinants of the polarity of plasma jets. The dynamics of the discharge reveal that the radial diffusion discharge is a special phenomenon observed only at the falling edge of the pulses. The radial transport of electrons, which is driven by the radial component of the applied electric field at the falling edge of the pulse, is shown to play an important role in increasing the seed electron density in the surrounding Ar. This result suggests a method to provide seed electrons at atmospheric pressure with a negative discharge. The polarity of the plasma jet is found to be determined by the pulse width rather than the polarity of the applied voltage, as it dictates the relative difference in the intensity of the two discharges in a single pulse, where the stronger discharge in a pulse dominates the behavior of the plasma jet. Accordingly, a method to control the polarity of a plasma jet through varying the pulse width is developed. Since plasma jets of different polarities differ remarkably in terms of their characteristics, the method to control the polarity reported in this paper will be of use for such applications as plasma-enhanced processing of materials and plasma biomedicine. Published by AIP Publishing. https://doi.org/10.1063/1.5010993

\section{INTRODUCTION}

Atmospheric pressure cold plasma jets have a variety of promising applications in biomedicine, environmental remediation, and material processing due to their simple structure and the ability to generate plasmas, which are not confined to a limited discharge gap. No surprisingly, in the last decade, plasma jets received a widespread attention, giving rise to a broad variety of plasma systems. In most situations, plasma jets are generated in ambient air, with $\mathrm{He}$ or $\mathrm{Ar}$ as the working gas. ${ }^{1,2}$ These plasmas are typically rich in reactive oxygen and nitrogen species, with a large number of active species such as $\mathrm{OH}, \mathrm{O}, \mathrm{NO}, \mathrm{NO}_{2}$, and $\mathrm{N}_{2}^{+}$arising from ambient air, as evidenced by the optical emission spectroscopy, Fourier transform infrared spectroscopy, and laser induced fluorescence. ${ }^{3-5}$ In some applications, such as decontamination, these active species are highly desirable since they play an important biological role, and thus largely

\footnotetext{
a) Author to whom correspondence should be addressed: yubin.xian@ hotmail.com
}

determine the outcome of the treatment. ${ }^{6-8}$ However, in other applications, e.g., material processing, they may not always make a positive contribution, which necessitates the use of certain measures to control the reactive species output of a plasma jet. ${ }^{9}$ Shielding gas is often applied in order to prevent the interactions between plasma effluent and ambient air. The chemical composition of the shielding gas has been suggested to affect the behavior of the plasma jet. ${ }^{10,11}$ This work seeks to explore the behavior of the plasma jet when propagating in a noble gas, thus in this paper He is adopted as the working gas, and Ar is used as the shielding gas. Radial propagation is observed at the falling edge of the discharge. In-depth study on this phenomenon provides a new insight into the polarity of the discharge in plasma jets, and a method with which the polarity of the plasma jet can be controlled is developed.

\section{EXPERIMENTAL SETUP}

As shown in Fig. 1, a pin-like electrode is placed in the center of the inner tube (inner diameter $4 \mathrm{~mm}$, outer 


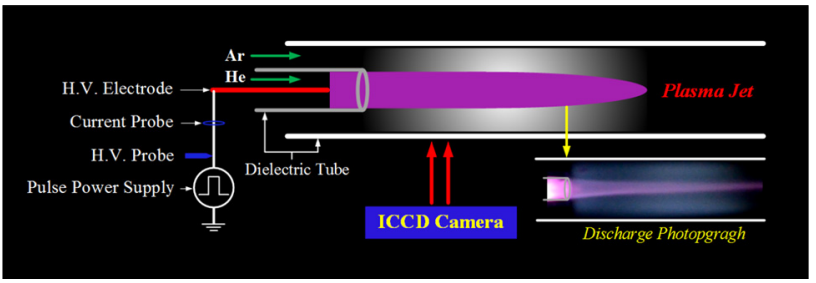

FIG. 1. Schematic view of the experimental setup. Inset depicts the photograph of the actual discharge generated using this experimental setup.

diameter $6 \mathrm{~mm}$ ), with the tip of the pin located $6 \mathrm{~mm}$ away from the exit. The radius of the high-voltage electrode and the curvature radius at the tip of the pin are both $200 \mu \mathrm{m}$. Working gas (He) flows in the inner tube at a flow rate of $1.5 \mathrm{~L} / \mathrm{min}$. An external gas flow (Ar) is applied through a concentric cylindrical channel (inner diameter $8 \mathrm{~mm}$ ) with a flow rate of $2.5 \mathrm{~L} / \mathrm{min}$. The plasma jet is generated in open air under ambient conditions. Thus, the ground can be considered at infinity. An ICCD camera (PI MAX II) is used to capture the dynamics of the propagation of the plasma jet. The frequency of the applied voltage is fixed at $8 \mathrm{kHz}$. The amplitude of the voltage is $5 \mathrm{kV}$ and $-5 \mathrm{kV}$ for positive and negative polarity, respectively. The pulse width is adjustable from $200 \mathrm{~ns}$ to $124.8 \mu$ s. The voltage on the high-voltage electrode is measured by a high-voltage probe (Tektronix P6015A), the current is determined by a current probe (Tektronix TCP202), and recorded by an oscilloscope (Tektronix DPO3034).

Figure 2 shows the voltage-current characteristics of the plasma jet driven by positive and negative pulses. The duration of the pulse is $1 \mu \mathrm{s}$, the frequency is $8 \mathrm{kHz}$, and the applied voltage is $5 \mathrm{kV}$ and $-5 \mathrm{kV}$ for the positive and negative pulses, respectively. It is evident that in the case of both positive and negative pulses, there are two discharges in a single pulse, one at the rising edge of the pulse, and the other at the falling edge. It should be pointed out that the current is the actual discharge current.

\section{EXPERIMENTAL RESULTS}

\section{A. The radial diffusion of the plasma jet in ambient $\mathrm{Ar}$}

The experimental results show that the obvious radial discharge could be observed when the plasma jet is operated using a certain pulse width. As shown in Fig. 3, when the plasma jet is driven by positive pulses, the diffusion plasma

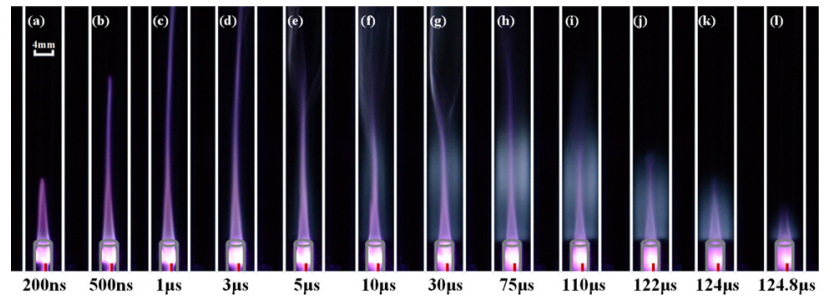

FIG. 3. Photographs of the plasma jet driven by positive pulses with different pulse width.

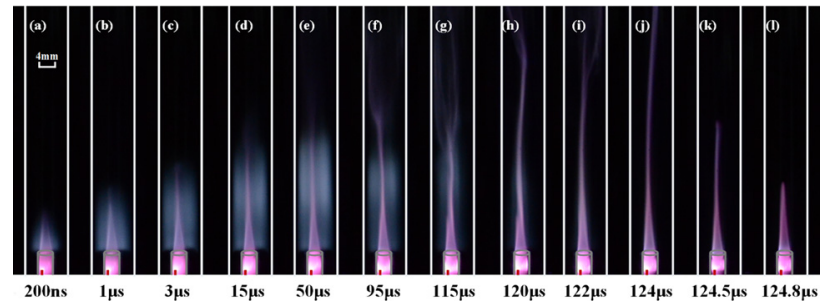

FIG. 4. Photographs of the plasma jet driven by negative pulses with different pulse width.

is obviously generated around the plasma jet when the pulse width is longer than $5 \mu \mathrm{s}$. This diffusion plasma is intensified and becomes brighter and larger as the pulse width is increased from $5 \mu$ s to $75 \mu \mathrm{s}$. However, the length of the plasma jet remains virtually constant. Further increases of the pulse width to $124.8 \mu \mathrm{s}$ ( $200 \mathrm{~ns}$ break) lead the diffusion plasma to become shorter and darker. At the same time, the length of the plasma jet also decreases.

On the other hand, the discharge driven by negative pulses shows the opposite behavior. As shown in Fig. 4, when the pulse width is increased from $200 \mathrm{~ns}$ to $50 \mu \mathrm{s}$, the length of the plasma jet increases and the diffusion plasma intensifies. When the pulse width is greater than $120 \mu \mathrm{s}$, the diffusion plasma disappears gradually.

Evidently, increasing the pulse width gives rise to opposite behavior in plasma jets driven by positive and negative pulses. When the sum of the widths of the positive and the negative pulses equals $125 \mu$ s (the pulse cycle), the same discharge phenomenon is observed, as shown in Figs. 3(h) and 4(e).

In addition, it attracts our attention that the diffusion plasma is homogeneous rather than filamentary. This is checked both by the naked eyes and the ICCD camera in a single shot. As we all know, Ar discharge is usually a filamentary
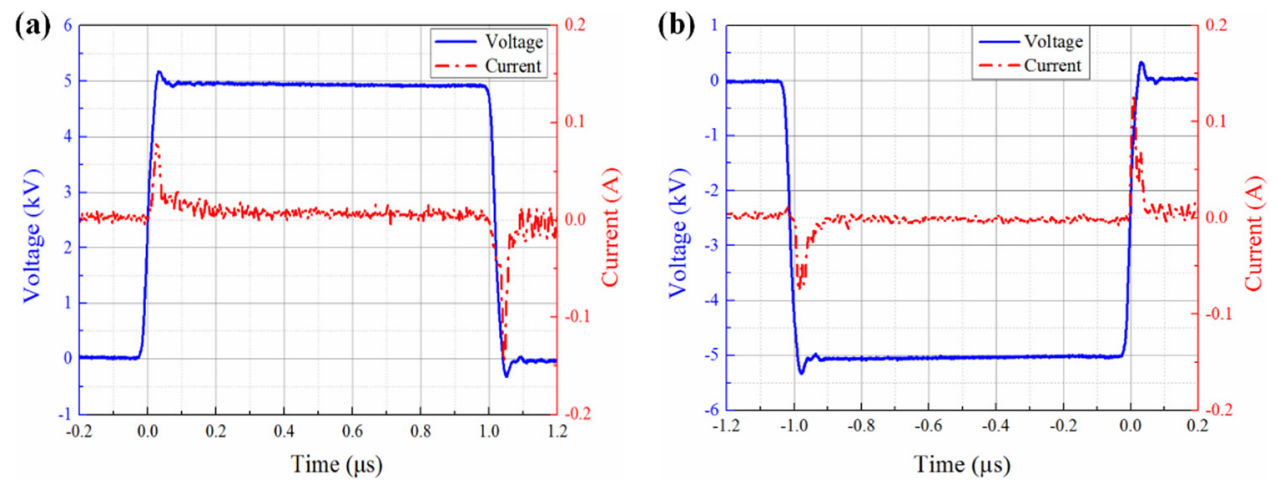

FIG. 2. Voltage-current characteristics of the discharge driven by (a) positive and (b) negative pulses. 


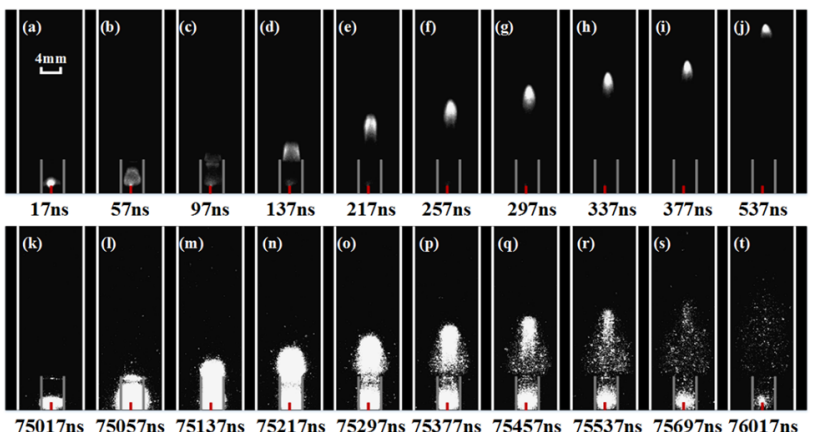

75017 ns 75057 ns $75137 n s$ 75217ns 75297 ns 75377 ns 75457 ns 75537 ns 75697 ns 76017 ns

FIG. 5. High-speed photograph of the plasma plume driven by positive pulses with a pulse width of $75 \mu \mathrm{s}$. The exposure time is fixed at $20 \mathrm{~ns}$. The time zero is the moment in time when the voltage begins to rise.

discharge, particularly at atmospheric pressure. But in this case, the plasma in $\mathrm{Ar}$ is as homogeneous as that at low pressure.

\section{B. The dynamics of the discharge driven by pulses of different polarities}

The results in Sec. III A show that the radial diffusion discharge is significantly associated with the pulse width. As we have seen, there are two discharges in a single pulse. The pulse width determines the time between these two discharges, and consequently affects the interaction between these two discharges. Visual observation with the naked eye does not permit to observe these individual discharges; thus, the dynamics of the discharge is acquired with an ICCD camera. When a positive pulse high voltage is applied, a plasma bullet is generated and propagates at the rising edge of the pulse, as shown in Figs. 5(a)-5(j). There is no difference from plasma jets generated in ambient air. However, at the falling edge of the pulse, the plasma bullet is much larger, and the discharge propagates both axially and radially, as shown in Figs. 5(k)-5(t). Thus, the plasma shows a cone shape. It is obvious that the radial diffusion discharge in Fig. 3 is induced by the discharge at the falling edge. When negative pules high voltage is applied, as shown in Fig. 6, the discharge behaves in a similar way to that observed when the positive pulse is used. A radial discharge is also observed at the falling edge of the pulse.

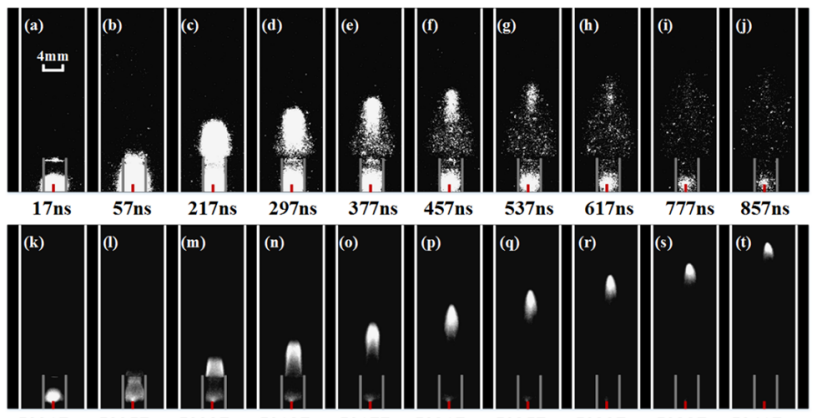

50017ns 50057ns 50097ns 50137ns 50177ns 50217ns 50257ns 50297ns 50337ns 50417ns

FIG. 6. High-speed photograph of the plasma plume for the negative pulse with a pulse width of $50 \mu \mathrm{s}$. The exposure time is fixed at $20 \mathrm{~ns}$. The time zero represents the moment in time when the voltage begins to fall.

\section{The radial diffusion characteristics for plasma jets operated using positive and negative pulses}

In order to have further understanding of the radial diffusion characteristics of thus-generated plasma jets, the minimum pulse width $p w_{\min }$ required for the radial discharge to form under various conditions is examined. In the case of positive pulse high voltage, the relationship between $p w_{\text {min }}$ and the voltage amplitude, the diameter of the outer tube, and the frequency is shown in Figs. 7 and 8. It is evident that the higher voltage leads to a smaller $p w_{\text {min }}$. When the voltage is $4 \mathrm{kV}$, with an $8 \mathrm{~mm}$ outer tube, $p w_{\text {min }}$ is approximately $3 \mu \mathrm{s}$. As the voltage increases to $9 \mathrm{kV}, p w_{\text {min }}$ linearly decreases to less than $1 \mu \mathrm{s}$. It is also revealed that a larger diameter of the outer tube requires larger minimum pulse width. Figure 8 shows that when the frequency changes from $4 \mathrm{kHz}$ to $9 \mathrm{kHz}, p w_{\text {min }}$ remains constant.

It should be noted that the data shown in Fig. 7 are somewhat different from those shown in Fig. 3. This is attributed to the differences in set-ups used to capture the images. In the case of Fig. 3, the specifics of the camera and the display of the monitor lead to partial loss of fine detail; thus, the actual $p w_{\text {min }}$ for the radial discharge is greater than that observed with a naked eye.

When a negative pulse high voltage is applied, the pulse width has a maximum width $p w_{\max }$ at which the radial discharge can be observed. As shown in Fig. 9, $p w_{\max }$ increases with the applied voltage, and decreases with the diameter of the outer tube. Similar to the case of the positive pulse, the discharge frequency has no influence on the radial discharge, as shown in Fig. 10.

\section{DISCUSSION}

\section{A. Mechanism of the radial discharge}

The radial discharge arises when the applied electric field is higher than the breakdown field strength in the surrounding region. Thus, both the increase in the applied

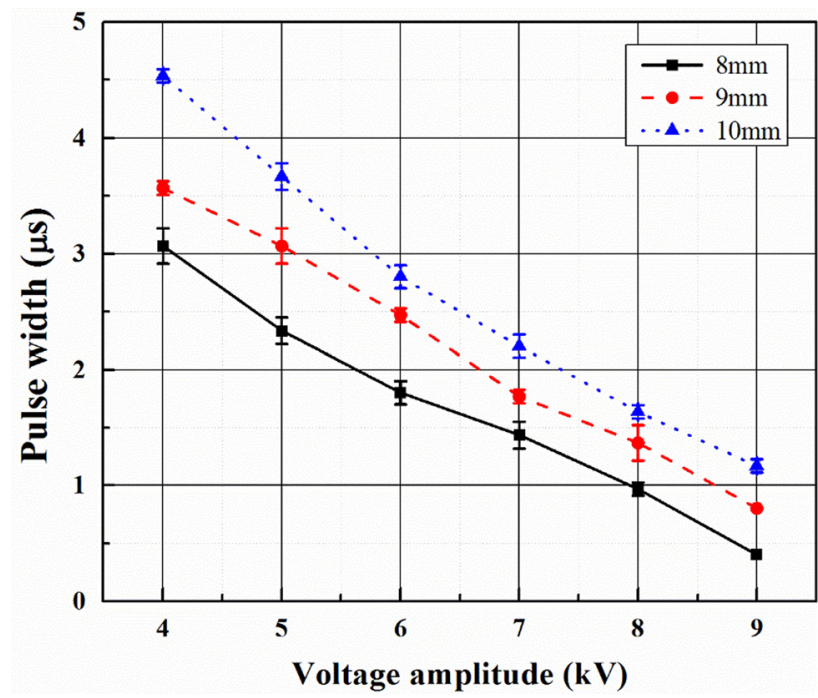

FIG. 7. Minimum pulse width $p w_{\text {min }}$ for the radial discharge driven by positive pulses with an amplitude varying from 4 to $9 \mathrm{kV}$. The diameter of the outer tube changes from 8 to $10 \mathrm{~mm}$. 


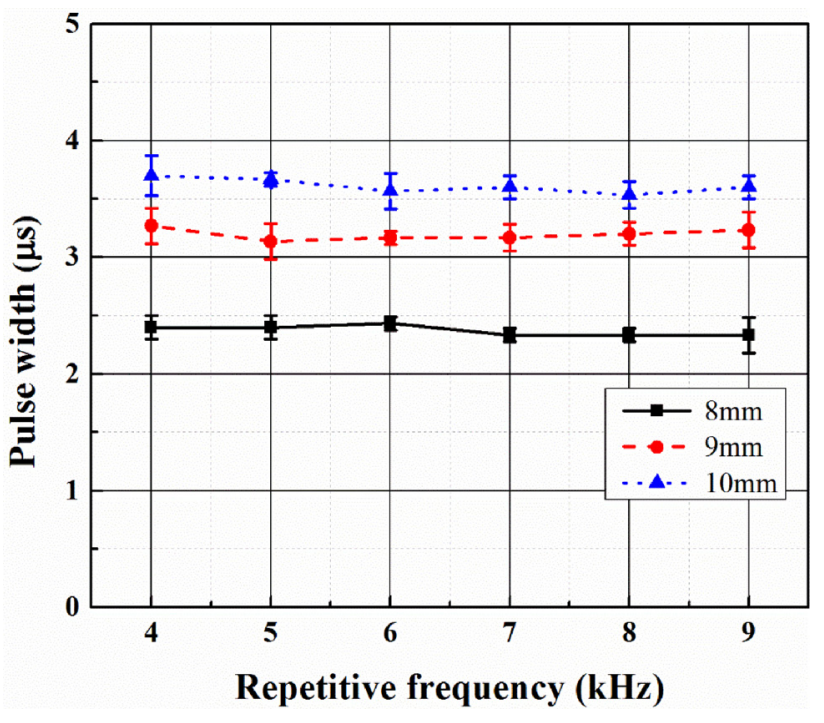

FIG. 8. Minimum pulse width $p w_{\text {min }}$ for the radial discharge generated in the outer tubes of various diameter $(8-10 \mathrm{~mm})$ driven by positive pulses as a function of frequency.

electric field and the decrease in the breakdown field strength could potentially induce the formation of the radial discharge. However, according to Ref. 12, the electric field is linked to the propagation speed of the plasma bullet. Since the speed of the plasma bullet is much lower for the falling edge discharge, the electric field for the falling edge discharge is lower than that for the rising edge discharge. Therefore, the radial discharge cannot be induced by increasing the applied electric field.

Therefore, the decrease in the breakdown field strength is the only cause to be responsible for the radial discharge. The breakdown voltage is largely determined by the $p d$ value, gas composition, and the density of seed electrons. Since the $p d$ value and the gas composition are kept constant in this study, seed electrons are likely to play a main role in reducing the breakdown voltage. In plasma jets, seed

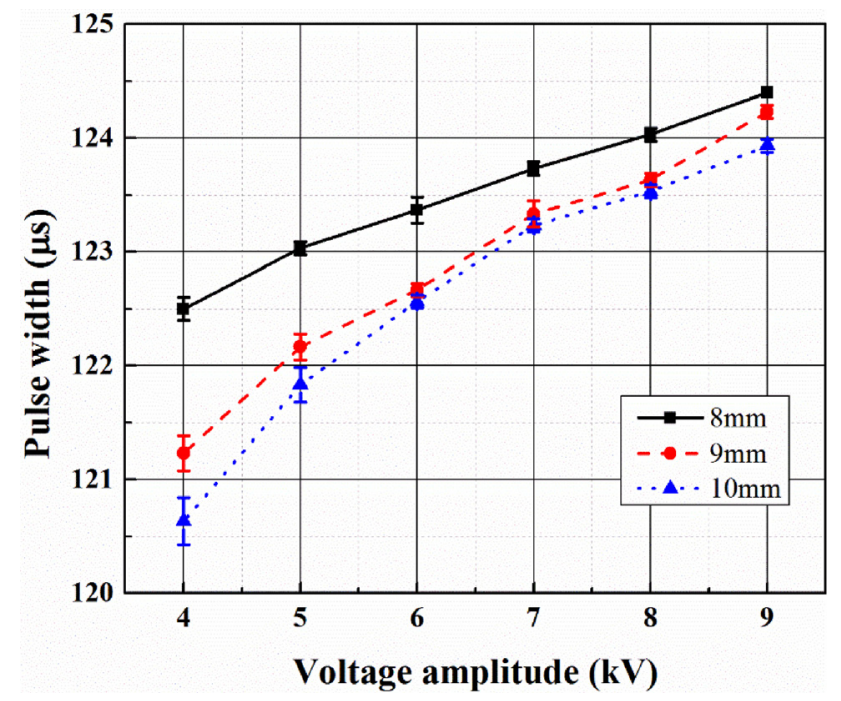

FIG. 9. Maximum pulse width $p w_{\max }$ for the radial discharge driven by negative pulses with an amplitude varying from 4 to $9 \mathrm{kV}$. The diameter of the outer tube changes from 8 to $10 \mathrm{~mm}$.

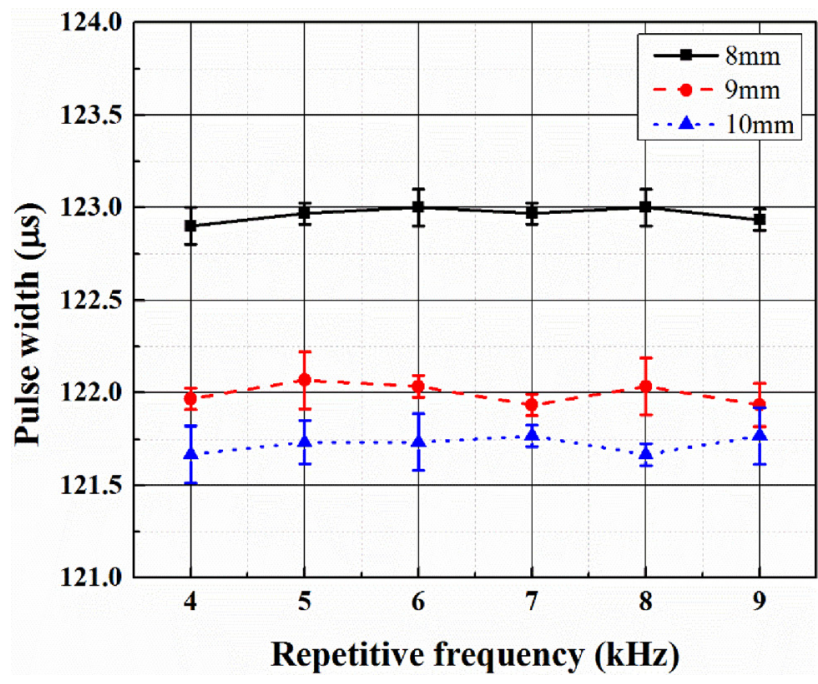

FIG. 10. Maximum pulse width $p w_{\max }$ for the radial discharge generated in the outer tubes of various diameter $(8-10 \mathrm{~mm})$ driven by negative pulses with different frequency.

electrons are generated in two ways, i.e., either through photoionization, or through the migration of electrons from the plasma to the ionization front, where they act as seed electrons. In the case of the former, since the discharge at the falling edge has a weaker electric field than the discharge at the rising edge, it cannot generate more photons or photoelectrons. The optical emission spectra confirm that no additional photons are generated by the discharge at the falling edge. ${ }^{13}$ Therefore, the increase in the number of seed electrons is more likely to result from the drifting of electrons in plasma. The question then arises as to why do electrons drift along the radial direction? Since the electric field is generated by a single pin electrode, it disperses with the distance from the electrode. The electric field contains both axial and radial components. As reported widely in literature, the axial directed electric field plays the main role in driving the plasma bullet to propagate. However, the role of the radial component of the electric field was ignored for a long time. Although the radial directed electric field has no direct effect on plasma bullet propagation, it affects the drift of electrons along the radial direction. When the pin electrode acts as an anode, the radial electric field drives the electrons to move from the ambient into the plasma jet, which increases the electron density in the so-called dark region. A dark region with a high electron density increases the electric field applied to the ionization front of the plasma bullet. As a result, the plasma bullet propagates faster and farther. On the other hand, when the pin electrode acts as a cathode, the radial component of the electric field drives the electrons from the plasma jet to the surrounding region. As a result, the surrounding region has a higher electron density. These electrons work as seed electrons to significantly reduce the breakdown electric field strength in this region. Although the breakdown strength of the electric field is not low enough to form a discharge in ambient air, the discharge can propagate radially in ambient Ar. Due to the high density of seed electrons and the reduced breakdown voltage, the radial discharge in ambient Ar is possible to be homogenous. But in 
the axial direction, the electric field is dispersed by the region between the electrode and the discharge front with lower electric density and a larger volume. Thus, the plasma bullet ignited from a cathode always propagates slower and to a shorter distance.

\section{B. Influence of pulse width on the polarity of the plasma jet}

We can find that a discharge ignited from an anode behaves differently from that ignited from a cathode. As discussed in Sec. IV A, this difference is due to the different direction of axial and radial electric fields. At the rising edge, the single electrode acts as an anode, so the discharge is a positive discharge (positive streamer). On the other hand, a negative discharge (negative streamer) is generated at the falling edge. Thus, the discharge observed by a naked eye is actually the overlap of positive and negative discharges. If the plasma jet behaves as a negative streamer, the rising edge discharge is much stronger than the falling edge discharge. Thus, the relative intensity of the positive discharge and negative discharge determines the polarity of the plasma jet.

The technical challenge is how to control the relative intensity of these two discharges. Several reports have shown that the plasma jet behaves significantly different when driven by positive and negative pulses. ${ }^{13-15}$ However, it is also commonly known that the polarity of the pulse is decided by the bias voltage. Decreasing the bias voltage can lead a positive pulse to become a negative one. Since the plasma jet device is equivalent to a capacitor, the bias voltage is filtered and does not directly influence the discharge. Yet, it is also evident that a positive plasma jet is achieved when driven by a positive pulse. This leads us to suggest that the polarity of the plasma jet is determined by the pulse width rather than anything else.

Since the discharge device is equal to a capacitor, the circuit is equal to an RC series circuit. The voltage across the capacitor is $U_{c}=U_{s}\left(1-e^{-t / \tau}\right)$. The rapidity with which $U_{c}$ increases is expressed in terms of the time constant $\tau=R C$. Thus, $U_{c}$ increases with $t$. In a pulse discharge, $U_{c}$ is the voltage applied on the electrode. The amplitude of $U_{c}$ is determined by the charge time $t$, which is the time between current and the preceding discharges. For a rising edge discharge, $t$ is the time between the falling edge of the preceding discharge and the rising edge of current discharge, seen as $t_{r}$ in Fig. 11. For a falling edge discharge, the charge time $t$ is equal to $t_{f}$ shown in Fig. 11. Thus, the voltage applied on

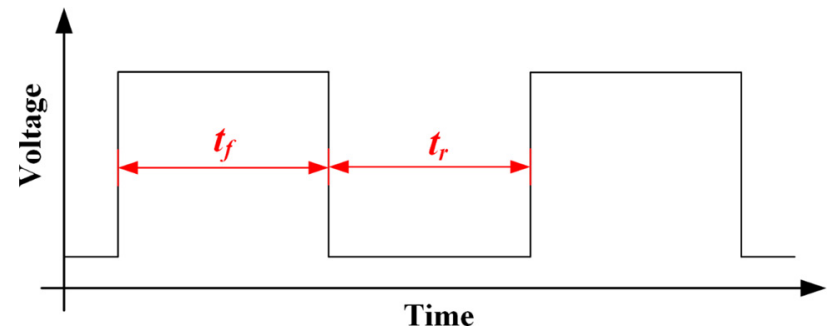

FIG. 11. Schematic for $t_{f}$ and $t_{r} . t_{f}$ is the time from the rising edge to the falling edge, and $t_{r}$ is the time from the falling edge to the rising edge. the electrode is determined by the pulse width. If $t_{r}$ is short, the rising edge discharge will be weak due to the low $U_{c}$. Therefore, a negative discharge plays a leading role, and the plasma jet behaves as a negative discharge. Instead, if $t_{f}$ is short, the rising edge discharge will dominate the plasma jet.

If $t_{r}$ and $t_{f}$ are kept unchanged, the polarity of the plasma jet will not change. This is the likely reason as to why the discharge driven by positive pulses with a duration of $75 \mu \mathrm{s}$ has the same phenomenon with that driven by negative pulses with a duration of $50 \mu \mathrm{s}$. In both of these two situations, $t_{r}=50 \mu \mathrm{s}, t_{f}=75 \mu \mathrm{s}$. It is therefore likely that the different polarities of the plasma jet driven by positive and negative pulses reported in Refs. 13-17 are actually induced by the different $t_{r}$ and $t_{f}$ rather than the polarity of the pulses.

According to Sec. III A, the duration between the falling edge and the rising edge has a significant effect on the radial diffusion of the discharge. This is likely to arise from the charge of the capacitor. If the amplitude of $U_{c}$ is high enough, the radial discharge occurs. This threshold voltage is expressed as $U_{c 0}$. If $t_{f}$ is long enough, $U_{c}$ will be charged to $U_{c o}$ before the falling edge discharge. As a result, the radial discharge is observed.

When the output voltage of the power supply $\left(U_{s}\right)$ increases, the time will be shorter for $U_{c}$ to rise to $U_{c 0}$. So $p w_{\text {min }}$ for the radial discharge will decrease accordingly. This is the likely reason for the decrease in $p w_{\text {min }}$ when the output voltage increases in Fig. 7 and for the increase in $p w_{\max }$ when the output voltage increases in Fig. 9.

The diameter of the outer tube impacts on the accumulation speed of space charge in the device because it is more difficult for space charge to run across a thicker Ar shielding gas layer. It is equivalent to increase in $R$ in the charge circuit. Thus, it takes longer time for $U_{c}$ to reach $U_{c 0}$. As a result, $p w_{\text {min }}$ in Fig. 7 increases when the diameter of the outer tube increases, and $p w_{\max }$ in Fig. 9 decreases when the diameter of the outer tube increases.

When the frequency is increased from $4 \mathrm{kHz}$ to $9 \mathrm{kHz}$, the pulse cycle changes from $250 \mu \mathrm{s}$ to $111 \mu \mathrm{s}$. While the minimum time needed for a radial discharge is as short as $5 \mu \mathrm{s}$ or less, which is negligible compared to the pulse cycle, it is expected that the frequency has no influence on the discharge, as shown in Figs. 8 and 10.

\section{CONCLUSION}

In this paper, the radial propagation of a plasma jet in ambient $\mathrm{Ar}$ is reported. The dynamics of the discharge reveals that it is a special phenomenon observed only in the falling edge discharge. Further study suggests that the radial drifting of the electron driven by the radial component of the applied electric field at the falling edge of the pulse plays an important role in increasing the seed electron density in the surrounding Ar. Therefore, a region with a low breakdown voltage is formed around the plasma jet. As a result, the radial diffusion discharge is observed at the falling edge of the pulse. In contrast, at the rising edge of the pulse, the seed electron density around the plasma jet is low due to the inward directed radial component of the electric field. Thus, the discharge cannot propagate radially. 
This result suggests a method to generate homogenous plasma at atmospheric pressure. A negative discharge in the center is used to provide seed electrons, and a uniform discharge of a large volume could be acquired in the ambient. Increasing the size and enhancing the uniformity of a discharge are highly desired for a variety of applications, including plasma medicine and materials processing.

This work demonstrates the radial drift of electron in plasma jets, and the quantitative study on it will be carried out in the future work. Furthermore, we believe that electron energy distribution in the rising and falling edge discharge could be different, and the study of electron energy distribution function (EEDF) is very important for a better understanding of the plasma bullet behaviour, and an extension of this effort from low-temperature plasmas $^{18,19}$ onto atmospheric-pressure plasma is an obvious opportunity for future research.

The results in Sec. III provide an illustration of an important mechanism for the control of the polarity of the plasma jet, which is shown to be determined by the pulse width rather than the polarity of the applied voltage. We show that there are two discharges in a single pulse, i.e., a positive discharge and a negative discharge. The pulse width determines the charge time before these two discharges, consequently, dictating the voltage across the discharge device. Therefore, the relative difference in the intensity between these two discharges in a pulse is dictated by the pulse width. Since the polarity of the plasma jet is defined by the stronger discharge in a pulse, we conclude that it is the pulse width that determines the polarity of the plasma jet.

Our findings provide a straightforward method to control the polarity of a plasma jet through varying the pulse width. Where a positive plasma jet is required, the duration from the falling edge to the rising edge should be much longer than that from the rising edge to the falling edge. To our knowledge, this is the only method to control the polarity of a plasma jet. Since plasma jets of different polarities have remarkable differences, the method to control the polarity introduced in this paper will be helpful in applications such as material and biomedicine.

\section{ACKNOWLEDGMENTS}

This work was supported by the National Natural Science Foundation of China (Grant No. 51507071).

${ }^{1}$ D. B. Graves, Phys. Plasmas 21(8), 080901 (2014).

${ }^{2}$ X. Lu, M. Laroussi, and V. Puech, Plasma Sources Sci. Technol. 21(3), 034005 (2012)

${ }^{3}$ X. Lu, G. V. Naidis, M. Laroussi, and K. Ostrikov, Phys. Rep.-Rev. Sect. Phys. Lett. 540(3), 123-166 (2014).

${ }^{4}$ X. P. Lu and S. Q. Wu, IEEE Trans. Plasma Sci. 41(8), 2313-2326 (2013).

${ }^{5}$ A. Schmidt-Bleker, J. Winter, S. Iseni, M. Duennbier, K. D. Weltmann, and S. Reuter, J. Phys. D: Appl. Phys. 47(14), 145201 (2014).

${ }^{6}$ D. B. Graves, Plasma Processes Polym. 11(12), 1120-1127 (2014).

${ }^{7}$ J. Winter, H. Tresp, M. U. Hammer, S. Iseni, S. Kupsch, A. SchmidtBleker, K. Wende, M. Duennbier, K. Masur, K. D. Weltmannan, and S. Reuter, J. Phys. D: Appl. Phys. 47(28), 285401 (2014).

${ }^{8}$ M. Ishaq, M. Evans, and K. Ostrikov, Int. J. Cancer 134(7), 1517-1528 (2014).

${ }^{9}$ I. Levchenko, K. Ostrikov, M. Keidar, and S. Xu, J. Appl. Phys. 98(6), 064304 (2005).

${ }^{10}$ Y. Xian, P. Zhang, X. Pei, and X. Lu, IEEE Trans. Plasma Sci. 42(10), 2448-2449 (2014).

${ }^{11}$ Y. B. Xian, D. D. Zou, X. P. Lu, Y. Pan, and K. Ostrikov, Appl. Phys. Lett. 103(9), 094103 (2013).

${ }^{12}$ X. Lu and M. Laroussi, J. Appl. Phys. 100(6), 063302 (2006).

${ }^{13}$ R. X. Wang, K. Zhang, Y. Shen, C. Zhang, W. D. Zhu, and T. Shao, Plasma Sources Sci. Technol. 25(1), 015020 (2016).

${ }^{14}$ Z. Xiong, X. Lu, Y. Xian, Z. Jiang, and Y. Pan, J. Appl. Phys. 108(10), 103303 (2010).

${ }^{15}$ T. Shao, W. J. Yang, C. Zhang, Z. Fang, Y. X. Zhou, and E. Schamiloglu, EPL 107(6), 065004 (2014).

${ }^{16}$ J. Jadidian, M. Zahn, N. Lavesson, O. Widlund, and K. Borg, IEEE Trans. Plasma Sci. 40(3), 909-918 (2012)

${ }^{17}$ C. Jiang, M. T. Chen, and M. A. Gundersen, J. Phys. D: Appl. Phys. 42(23), 232002 (2009).

${ }^{18}$ I. V. Schweigert, I. D. Kaganovich, and V. I. Demidov, Phys. Plasmas 20, 101606 (2013).

${ }^{19}$ B. Ramamurthi, D. J. Economou, and I. D. Kaganovich, Plasma Sources Sci. Technol. 12(3), 302-312 (2003). 